\title{
Minisymposium: Mathematics in Nanotechnology
}

\author{
Timothy G. Myers
}

\section{Description}

Nanotechnology is one of the key modern research directions, with billions being invested by governments throughout the world, and in particular by the US, Europe and Japan. Nanotechnology is relevant to a vast range of practical applications, such as in medicine, electronics, biomaterials and energy production. To date the vast majority of research has focussed on the experimental side, with the theory often lagging behind. However, there are a number of mathematical groups now working on topics relevant to the nano industry. In this minisymposium we intended to bring together a selection of speakers who discussed a broad range of topics relevant to nanoscience and who were able to demonstrate the relevance of mathematics to this research field.

The talks included in the minisymposium were the following:

- Tim Myers and Francesc Font (Centre de Recerca Matemàtica, Spain and University of Limerick, Ireland). Can you trust mathematics at the nanoscale?

- Helena Ribera (Centre de Recerca Matemàtica, Spain). Model for nanoparticle melting with a Newton cooling condition and size-dependent latent heat.

- Clemens Heitzinger (TU Wien, Austria). Stochastic partial differential equations for the modeling of nanowire and nanopore sensors.

- David Gómez-Castro (Complutense University of Madrid, Spain). Nanocatalysis: homogenization of critical scale two-phase composites with nonlinear reaction.

- Gary O'Keeffe (University of Limerick, Ireland). Modelling the efficiency of a nanofluid based direct absorption solar collector.

\footnotetext{
T.G. Myers $(\bowtie)$

Centre de Recerca Matemàtica, Campus de Bellaterra, Edifici C, 08193 Bellaterra, Barcelona, Spain

e-mail: tmyers@crm.cat
} 